ism. ${ }^{1011}$ Aortic valve homografts are, however, troublesome to procure and technically more difficult to insert than prostheses. They are not always haemodynamically perfect, and their longevity still awaits proof. During the last two years inverted aortic valve homografts have been put in the mitral ring after mounting on a metal frame, and autogenous fascia lata has been prepared in a similar fashion. The early results are encouraging; these valves also seem to confer freedom from embolic hazard, but the risk of late failure is still unknown. Thromboembolism can kill or maim, but haemodynamic failure is usually gradual and remediable. Many surgeons believe that this security from the tragedy of late coronary embolism puts the advantage firmly on the side of the homografts.

Even artificial valves are not immune from mechanical failure. A dehiscence may develop between the natural annulus and the sewing ring of the prosthesis. Regurgitation then gives rise to early postoperative haemodynamic deterioration and the frequent complication of mechanical haemolysis. ${ }^{12}$ Degenerative changes may distort the silicone rubber poppet of the caged aortic ball valve. ${ }^{13}$ This "variance" of the ball is a late complication that has only once been reported in the mitral valve, which is opened at lower pressure and therefore less violently. The variant ball becomes misshapen and discoloured; free mobility is lost so the ejection click gets softer, the ejection murmur louder, and the sounds may vary from beat to beat. A regurgitant murmur and mechanical haemolysis may follow, but dehiscence is distinguished from variance by its earlier onset. Both complications can be successfully corrected surgically.

A poor surgical result may also come about from failure of the myocardium despite a prosthesis working perfectly well. Much used to be said about "the myocardial factor," with the implication that surgical intervention might be delayed too long. While poor cardiac output inevitably leads to poor coronary blood flow (and the normally high oxygen extraction rate of the myocardium leaves little reserve for this) the main cause for postoperative myocardial failure may be myocardial damage consequent upon the operation itself. In Portland, U.S.A., N. R. Niles and J. R. Sandilands examined the hearts of 62 patients who had died at all stages up to six years after valve replacement. ${ }^{14}$ They found ischaemic necrosis or scars in 57 of the 62 cases. These were attributable to thrombosis on the prosthesis with coronary occlusion or embolism in 28 of the 36 late deaths. In 13 cases the infarcts

\footnotetext{
1 Herr, R. H., Starr, A., Pierie, W. R., Wood, J. A., and Bigelow, J. C., Annals of Thoracic Surgery, 1968, 6, 199.

Otto, T. J., Cleland, W. P., and Bentall, H. H., Fournal of Cardiovascular Surgery, 1969, 10, 339.

3 Morrow, A. G., Oldham, H. N., Elkins, R. C., and Braunwald, E., Circulation, 1967, 35, 962.

- Starr, A., Herr, R. H., and Wood, J. A., fournal of Thoracic and Cardiovascular Surgery, 1967, 54, 333.

s vascular Surgery, 1967, 54, 333. England fournal of Medicine, 1965, 273, 509.

- Braunwald, N. S., Ross, J., and Morrow, A. G., Circulation, 1967, 35, Suppl. No. 1, p. 63.

7 Duvoisin, G. E., Brandenburg, R. O., and McGoon, D. C., Circulation, 1967, 35, Suppl. No. 1, p. 70.

Yeh, T. J., Anabtawi, I. N., Cornett, V. E., and Ellison, R. G., Circulation, 1967, 35, Suppl. No. 1, p. 77.

- Sullivan, J. M., Harken, D. E., and Gorlin R., Circulation, 1969, 39, Suppl. No. 1, p. 149.

10 Barratt-Boyes, B. G., Lowe, J. B., Cole, D. S., and Kelly, D. T., Thorax, $1965,20,495$.

11 Ross, D., Surgery, 1968, 63, 382.

12 Kastor, J. A., et al., Fournal of Thoracic and Cardiovascular Surgery, 1968, 56, 279.

13 Bonnabeau, R. C., and Lillehei, C. W., fournal of Thoracic and Cardiovascular Surgery, 1968, 56, 258.

14 Niles, N. R., and Sandilands, J. R., Diseases of the Chest, 1969, 56, 373.

15 Roberts, W. C., and Morrow, A. G., Archives of Pathology, 1966, 82, 164.

16 Braniff, B. A., Shumway, N. E., and Harrison, D. C., New England Fournal of Medicine, 1967, 276, 1464.
}

appeared temporally related to the operation: these tended to be haemorrhagic, patchy, or miliary and most heavily affected the inner layers of the left ventricular walls. These important observations have clear bearing on the once common postoperative low-output syndrome, which has now become rare even in high-risk cases. The improvement is attributable to better control of coronary perfusion and to prevention of air and particle embolism.

Infection on artificial prostheses is another major problem. Infection which is acquired at the time of surgery is likely to be due either to a resistant hospital staphylococcus or to a ubiquitous organism of low pathogenicity, which is often almost equally hard to treat. Positive blood cultures may not be obtained because of the broad-spectrum antibiotics usually prescribed to "cover" surgery, yet early diagnosis is particularily vital in these cases. Later infections are caused by similar organisms to those which infect diseased natural valves, and are therefore most commonly due to Streptococcus viridans in patients with teeth and Gram-negative bacteria in elderly patients with disease of the gastrointestinal or urinary tracts. Fungus endocarditis is particularly sinister. The spores probably enter the heart at the time of surgery, but their growth is so insidious, the constitutional reaction often so slight, and the incidence of fungi in the blood stream likely to be so sporadic that recognition may be delayed for many months. Infection in a prosthesis is a very grave complication and in some cases surgical excision may offer the only chance of a cure. ${ }^{15} 16$

Notwithstanding this formidable list of potential hazards the chances of a given patient with an artificial valve avoiding trouble are steadily improving. The operative risk still lies between $5 \%$ and $25 \%$, but at present we can expect that up to $70 \%$ of the survivors of cardiac valve replacement will be alive, well, and able to work five years after surgery. It is reasonable to hope that if a patient has avoided embolism, infection, and mechanical breakdown over this length of time that the prosthesis will be so well endothelialized and incorporated that the chances of the next 5 or 50 years being as good are even brighter.

\section{Haematuria in Childhood}

The causes of haematuria in childhood include structural abnormalities of the renal tract such as hydronephrosis, polycystic kidney, and tumours of the kidney or bladder; stones and crystals; trauma; meatal ulcer; infections caused by bacteria (including tuberculosis), viruses, or schistosomata; acute and chronic glomerulonephritis; Henoch-Schoenlein syndrome; haematological disorders such as sickle-cell anaemia and leukaemia; haemorrhagic disorders including haemophilia and scurvy; systemic diseases such as endocarditis and disseminated lupus; and certain drugs and poisons. Lists of this kind are of very limited use to the clinician: they suggest unreal diagnostic dilemmas and bemuse those who try to remember them. In practice a child who presents with haematuria as the main problem is most likely to have acute glomerulonephritis, urinary infection, renal trauma, or the well-recognized but still puzzling disorder of recurrent haematuria, discussed by Drs. E. F. Glasgow, M. W. Moncrieff, and R. H. R. White at page 687 of this week's B.M.F. A rational scheme of investigation for diagnosis of the commoner causes will also disclose most of the rarer ones.

The urine must first be examined to confirm that the discoloration is due to blood. The simplest test is Hemastix or .
}


Occultest, but these do not distinguish haematuria from haemoglobinuria. Microscopy of fresh urine is far more informative. It confirms the presence of red cells and may also show granular or red-cell casts, indicating that the bleeding is from the kidney rather than lower in the urinary tract; or organisms may be seen, indicating bacterial infection. The degree of mixing of blood and urine is not usually a helpful physical symptom.

Acute glomerulonephritis remains the commonest cause of haematuria in childhood. ${ }^{12}$ It can usually be diagnosed clinically from the sudden onset, with oedema and oliguria, and the history suggesting preceding streptococcal upper respiratory infection. Culture of $\beta$-haemolytic streptococci from the throat or a raised antistreptolysin titre supports the diagnosis. Provided the child does not have severe oliguria or distinct hypertension, acute glomerulonephritis can reasonably be managed at home. ${ }^{3}$

Patients with all other forms of haematuria not due to an obvious local cause like meatal ulcer should probably be referred to hospital in the first attack, even if, as in cases of renal trauma and Henoch-Schoenlein syndrome, the diagnosis seems certain clinically. The immediate investigations should generally include urine analysis, microscopy, and culture, throat swab, full blood count, blood urea, antistreptolysin titre, serum $\beta_{1 \mathrm{c}}$-globulin ${ }^{4-6}$ (if possible), bleeding and clotting tests, sickling test if the child is a Negro, abdominal $x$-ray, and tuberculin skin test. Of these the urine culture is the most likely to provide the diagnosis, for urinary infection is the second commonest cause of childhood haematuria. ${ }^{3}$ Further investigation and treatment of proved bacterial infection must be thorough. ${ }^{78}$ There is also a viral form of acute haemorrhagic cystitis in children, adenovirus type 11 being the cause, ${ }^{9}$ and investigations into that are worthwhile if they are possible.

An intravenous pyelogram is indicated next in all cases not clearly due to acute glomerulonephritis. However, it will be normal in most cases of haematuria in childhood. Though conventional surgical investigation includes cystoscopy and retrograde pyelography, these are not necessarily appropriate in a child, in whom the common causes are mainly "medical." In fact, if the investigations so far have not established the cause of bleeding, it is likely to be the condition variously

1 McConville, J. M., West, C. D., and McAdams, A. J., Fournal of Pediatrics, 1966, 69, 207.

Harrison, W. E., Habib, H. N., Smith, E. I., and McCarthy, R. P. fournal of Urology, 1966, 96, 95

3 Arneil, G. C., British Medical fournal, 1965, 1, 1479

Gotoff, S. P., Fellers, F. X., Vawter, G. F., Janeway, C. A., and Rosen, F. S., New England fournal of Medicine, 1965, 273, 524.

5 Ogg, C. S., Cameron, J. S., and White, R. H. R., Lancet, 1968, 2, 78.

- Gotoff, S. P., Isaacs, E. W., Muehrcke, R. C., and Smith, R. D., Annal of Internal Medicine, $1969, \mathbf{7 1}, 327$.

Smellie, J. M., Hodson, C. J., Edwards, D., and Normand, I. C. S., British Medical fournal, 1964, 2, 1222.

- Smellie, J. M., and Normand, I. C. S., Proceedings of the Royal Society of Medicine, 1966, 59, 415 .

- Numazaki, Y., et al., New England fournal of Medicine, 1968, 278, 700

10 Numazaki, Y., et al., New England fournal of Medicine, 1968, 278, 700. Bodian, M., Black, J. A., Kobayashi, N., Lake,

11 Ferris, T. F., Gorden, P., Kashgarian, M., and Epstein, F. H., New England fournal of Medicine, 1967, 276, 770.

12 Ross, J. H., Quarterly fournal of Medicine, 1960, 29, 391

13 Travis, L. B., Daeschner, C. W., Dodge, W. F., Hopps, H. C., and Rosenberg, H. S., Fournal of Pediatrics, 1962, 60, 24.

14 Livaditis, A., and Éricsson, N. O., Acta Paediatrica, 51, 630.

15 Wyllie, G. G., Proceedings of the Royal Society of Medicine, 1955, 48, 1113 .

16 Todd, R. McL., and Bouton, M. J., Proceedings of the Royal Society of Medicine, 1966, 59, 209.

17 Singer, D. B., Hill, L. L., Rosenberg, H. S., Marshall, J., and Swenson,

18 Arneil, G. C., Lam, C. N., McDonald, A. M., and McDonald, M., British Medical fournal, 1969, 2, 233.
.

19 Johnston, C., and Shuler, S., Archives of Disease in Childhood, 1969, 44, 483.

${ }^{20}$ Perkoff, G. T., New England fournal of Medicine, 1967, 277, 79 and 129.

21 Williamson, D. A. J., Lancet, 1961, 2, 1321. described as focal nephritis, ${ }^{10-12}$ or benign, ${ }^{1}$ idiopathic, ${ }^{13}$ essential, ${ }^{14}$ or recurrent haematuria. ${ }^{15-19}$ This is commoner in males than females and may begin at any time in childhood or early adult life. There are attacks of gross haematuria, usually lasting less than a week, often precipitated by nonstreptococcal upper respiratory infection and less often by exercise. There may be abdominal pain, but oedema, oliguria, and hypertension are absent. Red-cell and granular casts are often present in the urine, and proteinuria and microscopical haematuria may persist between attacks. Though haematuria may be familial, 17 most cases do not fall among those hereditary renal diseases, ${ }^{20}$ such as Alport's syndrome, ${ }^{21}$ which have a bad prognosis, especially in males. The histological appearances reported from renal biopsies in recurrent haematuria have varied, partly, as Glasgow and his colleagues show, owing to differences in terminology. In their own series, however, the histological appearance ranged from virtually normal to that of proliferative glomerulonephritis, the latter being more likely if there was persistent proteinuria.

Recurrent haematuria is evidently a mixed bag pathologically, and it is unwise to be too dogmatic about prognosis. ${ }^{17} 18$ But the papers cited have been generally reassuring. They refer to over 300 patients, most of whom are known to have remained healthy, many for well over five years. 11215 17-19

Cystoscopy and retrograde pyelography contribute nothing in this condition, and it seems reasonable to omit them from the routine investigation of a child with haematuria, though they should be considered if bleeding is severe, prolonged, or otherwise unusual and unexplained. Renal biopsy is much more likely to show an abnormality, and histological information like that given by Glasgow and colleagues, combined with follow-up after biopsy, ${ }^{19}$ seems the most promising way of sorting out "recurrent haematuria" and establishing criteria for prognosis. But at present, though biopsy shows the degree of histological abnormality, it has not been unequivocally proved to help assess prognosis, and it does not influence treatment. It cannot, therefore, be considered a necessary routine investigation in a child with unexplained haematuria. This would no longer be true if some patients with recurrent haematuria were shown to have progressive renal lesions, recognizable on biopsy, which responded to immunosuppressive therapy.

\section{The "Empty" Sella}

Occasionally the sella turcica is found to contain less pituitary tissue than usual, the space above the remnant being replaced by cerebrospinal fluid. W. Busch ${ }^{1}$ found "empty" sellas in $5.5 \%$ of 788 patients examined post mortem. Other observers $^{2-4}$ have reported a similar incidence.

The radiological measurement of the antero-posterior length and the width of a normal pituitary gland is reasonably accurate, but the height sometimes presents a problem. ${ }^{5}$ Thus the upper surface of the pituitary may be several millimetres below the expected level, and is then well described ${ }^{6}$ by the non-committal term "empty" sella. Failure to recognize this abnormality in patients being treated by radiologically controlled irradiation destruction of the pituitary would lead to a higher degree of damage to the upper surface of the gland than intended. The consequence could be leakage of cerebrospinal fluid and perhaps even damage to a low-placed optic chiasma. Fortunately the degree of depression is rarely severe if the sella appears normal radiologically and the patient is without clinical features of pituitary disease. ${ }^{7}$ Usually the depression is greatest 